\title{
Classical nature of nuclear spin noise near clock transitions of Bi donors in silicon
}

\author{
Wen-Long Ma, ${ }^{1,2,3}$ Gary Wolfowicz, ${ }^{4,5}$ Shu-Shen Li, ${ }^{1,3}$ John J. L. Morton, ${ }^{4,6,{ }^{*}}$ and Ren-Bao Liu ${ }^{2,7,8, \dagger}$ \\ ${ }^{1}$ State Key Laboratory of Superlattices and Microstructures, Institute of Semiconductors, Chinese Academy of Sciences, Beijing 100083, China \\ ${ }^{2}$ Department of Physics, The Chinese University of Hong Kong, Shatin, N.T., Hong Kong, China \\ ${ }^{3}$ Synergetic Innovation Center of Quantum Information and Quantum Physics, University of Science and Technology of China, \\ Hefei, Anhui 230026, China \\ ${ }^{4}$ London Centre for Nanotechnology, University College London, London WC1H OAH, United Kingdom \\ ${ }^{5}$ Department of Materials, Oxford University, Oxford OX1 3PH, United Kingdom \\ ${ }^{6}$ Department of Electronic and Electrical Engineering, University College London, London WC1E 7JE, United Kingdom \\ ${ }^{7}$ Centre for Quantum Coherence, The Chinese University of Hong Kong, Shatin, N.T., Hong Kong, China \\ ${ }^{8}$ Institute of Theoretical Physics, The Chinese University of Hong Kong, Shatin, N.T., Hong Kong, China
}

(Received 19 May 2015; revised manuscript received 13 September 2015; published 8 October 2015)

\begin{abstract}
Whether a quantum bath can be approximated as classical Gaussian noise is a fundamental issue in central spin decoherence and also of practical importance in designing noise-resilient quantum control. Spin qubits based on bismuth donors in silicon have tunable interactions with nuclear spin baths and are first-order insensitive to magnetic noise at so-called clock transitions (CTs). This system is therefore ideal for studying the quantum/classical Gaussian nature of nuclear spin baths since the qubit-bath interaction strength determines the back-action on the baths and hence the adequacy of a Gaussian noise model. We develop a Gaussian noise model with noise correlations determined by quantum calculations and compare the classical noise approximation to the full quantum bath theory. We experimentally test our model through a dynamical decoupling sequence of up to 128 pulses, finding good agreement with simulations and measuring electron spin coherence times approaching $1 \mathrm{~s}-$ notably using natural silicon. Our theoretical and experimental study demonstrates that the noise from a nuclear spin bath is analogous to classical Gaussian noise if the back-action of the qubit on the bath is small compared to the internal bath dynamics, as is the case close to CTs. However, far from the CTs, the back-action of the central spin on the bath is such that the quantum model is required to accurately model spin decoherence.
\end{abstract}

DOI: 10.1103/PhysRevB.92.161403

PACS number(s): 05.30.-d, 03.65.Yz, 76.30.Mi, 76.60.Lz

Introduction. Central spin decoherence due to coupling to the environment is not only a central issue in understanding quantum-to-classical transitions [1,2], but also one of the key challenges in the realization of quantum computation [3]. There are two distinct models to describe the decoherence processes in such cases: in the semiclassical model, the central spin accumulates random phases due to thermal or quantum fluctuations of the environment [4,5], while in the quantum model, the coupling between the central spin and the environment produces entanglement and results in leakage of the which-way information from the central spin to the environment [6-9]. The fundamental difference between these two models lies in the fact that the classical noise is independent of the central spin state while the quantum noise is governed by the difference of environmental Hamiltonians conditioned on the qubit state, called the back-action from the central spin $[10,11]$.

The classical noise model of quantum baths, especially the Gaussian stochastic noise model, is a useful approximation in designing noise-resilient quantum control [12], which would otherwise require a large amount of numerical simulations of many-body dynamics of quantum baths. Dynamical decoupling has been employed to extract the noise spectra of baths [13-17], which are in turn used to design optimal quantum control for protecting quantum coherence and quantum gates. The viability of such methods critically

\footnotetext{
*jjl.morton@ucl.ac.uk
}

†rbliu@phys.cuhk.edu.hk depends on whether the Gaussian noise picture is valid or not. Therefore, examining the conditions for the validity of a classical Gaussian noise model is not only of fundamental interest but also highly desirable for accurate quantum control under realistic conditions.

Bismuth donors in silicon $(\mathrm{Si}: \mathrm{Bi})$ have recently attracted much attention in spin-based quantum computation due to a number of favorable properties [18-21]. These include long electron spin coherence times of up to $3 \mathrm{~s}$ [22] observed for $\mathrm{Bi}$ donors (in isotopically enriched silicon-28) tuned to so-called clock transitions (CTs) - also known as optimal working points [23] or zero first-order Zeeman transitions)whose frequency is insensitive, to first order, to magnetic field fluctuations. The coherence times of donor electron spins in natural silicon are typically limited to a few hundred microseconds by the $5 \%$ naturally abundant ${ }^{29}$ Si nuclear spins. At CTs, the effect of the ${ }^{29} \mathrm{Si}$ on the electron spin coherence is strongly suppressed (though not completely removed), leading to coherence times of up to $100 \mathrm{~ms}$. Previous studies have focused on quantum approaches to model electron spin decoherence from ${ }^{29} \mathrm{Si}$ nuclear spin baths [24,25]; however, performing such calculations near the CTs can be challenging due to the strongly correlated nuclear spin baths [24].

In this Rapid Communication, we explore the applicability of a classical Gaussian stochastic noise description [26,27] of the nuclear spin bath around $\mathrm{Bi}$ donors in natural silicon, especially near the Si:Bi CTs. Such classical Gaussian noise is fully characterized by the two-point correlation functions or the noise spectrum [27]. We demonstrate the validity of such a semiclassical model by comparisons with exact 
results from the quantum model and with experimental measurements.

System and Hamiltonian. For the Si:Bi system interacting with a ${ }^{29} \mathrm{Si}$ nuclear spin bath $\left(I_{i}=1 / 2\right.$ and natural abundance $4.7 \%$ throughout the host lattice), the system Hamiltonian is divided into three parts [24,25]:

$$
H=H_{\mathrm{cs}}+H_{\text {int }}+H_{\text {bath }},
$$

with

$$
\begin{aligned}
H_{\mathrm{cs}} & =\omega_{e} S^{z}-\omega_{n}^{\mathrm{Bi}} I_{0}^{z}+A_{0} \mathbf{S} \cdot \mathbf{I}_{0}, \\
H_{\mathrm{int}} & =S^{z} \sum_{i} A_{i} I_{i}^{z}, \\
H_{\mathrm{bath}} & =-\omega_{n}^{\mathrm{Si}} \sum_{i} I_{i}^{z}+\sum_{i<j} \mathbf{I}_{i} \cdot \mathbb{D}_{i j} \cdot \mathbf{I}_{j},
\end{aligned}
$$

where $\mathbf{S}$ is the Bi donor electron spin operator, $\mathbf{I}_{0}\left(\mathbf{I}_{i}\right)$ is the ${ }^{209} \mathrm{Bi}\left({ }^{29} \mathrm{Si}\right)$ nuclear spin operator, $\omega_{\mathrm{e}}, \omega_{\mathrm{n}}^{\mathrm{Bi}}$, and $\omega_{\mathrm{n}}^{\mathrm{Si}}$ are correspondingly the Larmor frequencies of the donor electron spin, ${ }^{209} \mathrm{Bi}$ and ${ }^{29} \mathrm{Si}$ nuclear spins (which are related to their gyromagnetic ratios $\gamma$ by $\left.\omega_{\alpha}=\gamma_{\alpha} B\right), A_{0}\left(A_{i}\right)$ is the coupling strength between the donor electron spin and the ${ }^{209} \mathrm{Bi}$ $\left({ }^{29} \mathrm{Si}\right)$ nuclear spins, $\mathbb{D}_{i j}$ is the nuclear-nuclear interaction tensor, and $B$ is the magnetic field applied along the $z$ axis. Here we have neglected the nonsecular terms in $H_{\text {int }}$ which induce the central spin relaxation, because the qubit energy splitting is much larger than the qubit-bath coupling (see the Supplemental Material for detailed discussions on the effects of the nonsecular terms on the pure-dephasing model) [28].

Semiclassical model for quantum decoherence. The combined $\mathrm{Bi}$ donor electron-nuclear spin system $(S=1 / 2$, and $I_{0}=9 / 2$ ) with the Hamiltonian $H_{\mathrm{cs}}$ has 20 eigenstates with eigenenergies dependent on the magnetic field [28]. By focusing on a pair of the eigenstates, $|+\rangle$ and $|-\rangle$, of the central spin, we can recast the system Hamiltonian in Eq. (1) as a function of these two central spin states:

$$
H^{( \pm)}= \pm \frac{P_{+}-P_{-}}{2} \beta^{z}+\frac{P_{+}+P_{-}}{2} \beta^{z}+H_{\text {bath }},
$$

where $\beta^{z}=\sum_{i} \beta_{i}^{z}=\sum_{i} A_{i} I_{i}^{z}$ is the Overhauser field operator and $P_{ \pm}=\left\langle \pm\left|S^{z}\right| \pm\right\rangle$. The CTs are characterized by $P_{+} \simeq P_{-}$ such that central spin decoherence at the $|+\rangle \leftrightarrow|-\rangle$ transition is strongly suppressed due to the nearly identical evolutions of the nuclear environment conditioned on the central spin state (i.e., $H^{(+)} \simeq H^{(-)}$). Consequently, the back-action of the central spin on the environment $[10,11]$ is quite small near the CTs $\left(\left|P_{+}-P_{-}\right| \ll\left|P_{+}+P_{-}\right|\right)$, so we may infer that a semiclassical model for quantum decoherence should well reproduce the results from the quantum model.

The two-point correlation function of the nuclear spin noise is defined as

$$
C(t)=\left\langle\beta^{z}(t) \beta^{z}(0)\right\rangle=\left\langle e^{i H_{e} t} \beta^{z}(0) e^{-i H_{e} t} \beta^{z}(0)\right\rangle,
$$

where $\langle\cdots\rangle=\operatorname{Tr}\left[\rho_{b} \cdots\right]$ denotes the ensemble average over the density matrix $\rho_{b}=\mathbb{I} / 2^{M}$ for $M$ nuclear spins at infinite temperature, and $H_{e}=\frac{\left|P_{+}\right|+\left|P_{-}\right|}{2} \beta^{z}+H_{\text {bath }}$ is the effective Hamiltonian for the nuclear spin bath. We include the term $\frac{\left|P_{+}\right|+\left|P_{-}\right|}{2} \beta^{z}$ in $H_{e}$ for the following considerations. When the qubit is in the state $|+\rangle$ or $|-\rangle$, the nuclear spin bath feels a bias field $P_{ \pm} \beta^{z}$, which significantly affects the bath dynamics and hence the correlation functions. Note that the sign of the bias field is immaterial, that is, the bias field with opposite sign (i.e., $-P_{ \pm} \beta^{z}$ ) would produce the same bath correlation function. For the qubit in a superposition state, to average the effects of different bias fields due to different qubit states, we choose the form $\frac{\left|P_{+}\right|+\left|P_{-}\right|}{2} \beta^{z}$. The numerical results actually show that such a choice yields the least discrepancy between calculation and measurement.

In dynamical decoupling (DD) control, a sequence of $N \pi$ flips are applied to the central spin at times $\left\{t_{1}, t_{2}, \ldots, t_{N}\right\}$ to suppress magnetic noise [29,30]. In this Rapid Communication we consider $N$-pulse Carr-Purcell-Meiboom-Gill $(\mathrm{CPMG}-N)$ control [31,32] with $t_{k}=(2 k-1) t /(2 N)$ (for $k=1,2, \ldots, N)$. With the Gaussian approximation [27], the central spin decoherence under DD control is

$$
\begin{aligned}
L(t) & =\exp \left[-\frac{P_{e}^{2}}{2} \int_{0}^{t} \int_{0}^{t} d t_{1} d t_{2} C\left(t_{1}-t_{2}\right) f\left(t_{1}\right) f\left(t_{2}\right)\right] \\
& =\exp \left[-\frac{P_{e}^{2}}{2} \int_{-\infty}^{\infty} \frac{d \omega}{2 \pi} C(\omega) \frac{F(\omega, t)}{\omega^{2}}\right],
\end{aligned}
$$

where $P_{e}=\left|P_{+}-P_{-}\right|$is the scaling factor, and $C(\omega)=$ $\int_{-\infty}^{\infty} e^{i \omega t} C(t) d t$ is the noise spectrum of the nuclear spin noise [since $C(t)=C(-t)$, so $C(\omega)=C(-\omega)], f(t)=(-1)^{k}$ for $\left[t_{k-1}, t_{k}\right]$ is the modulation function $\left(t_{0}=0, t_{N+1}=t\right)$, and $F(\omega, t)=\left|\sum_{k=0}^{N}(-1)^{k}\left(e^{i \omega t_{k+1}}-e^{i \omega t_{k}}\right)\right|^{2}$ is the filter function.

Characterization of the nuclear spin noise. The correlation functions $C(t)$ for the natural ${ }^{29} \mathrm{Si}$ nuclear spin bath in silicon are calculated by the cluster-correlation expansion (CCE) method $[8,12,28]$. As a typical example, we choose to study the central spin transition between $|+\rangle \equiv|5,-1\rangle$ and $|-\rangle \equiv|4,-2\rangle$, using the basis $\left|F, m_{F}\right\rangle$ where $F\left(=I_{0} \pm S\right)$ is the total spin and $m_{F}\left(=m_{S}+m_{I_{0}}\right)$ is its projection. This transition is a CT at $B=79.9 \mathrm{mT}$ (where $P_{+}=P_{-}=0.0525$ ).

In Fig. 1(a), we show the relative correlation function $C(t)-C(0)$ corresponding to the quantum fluctuations of nuclear spin noise calculated for a random nuclear spin configuration with B $\|[110]$. Since $C(t)$ depends on the specific nuclear spin configuration, we show in Fig. 1(b) the relative correlation functions averaged over many bath configurations. The results can be well fitted by a stretched exponential decay:

$$
C(t)=C(0)+\Delta^{2}\left\{\exp \left[-(|t| / \tau)^{n}\right]-1\right\},
$$

where $\Delta$ is the correlation amplitude, $\tau$ is the correlation time, and $n$ is the stretch factor. In Fig. 1(c), we show the fitting parameters as functions of the magnetic field orientation. As the field direction varies from [001] $\rightarrow[111] \rightarrow[110]$, the correlation amplitude $\Delta$ first increases with $\theta$ (the angle from [001]), reaches a maximum at about $55^{\circ}$ ([111]), and then slightly decreases. The stretch factor $n$ has about the same trend as $\Delta$ apart from some oscillations due to systematic fitting errors, while the correlation time $\tau$ has the opposite trend. The dependence of the correlation functions on the magnetic field orientation is due to the anisotropy of the dipolar interaction between nuclear spins and the silicon lattice structure [33,34], and can be well understood from a microscopic analysis of the nuclear spin dynamics. 

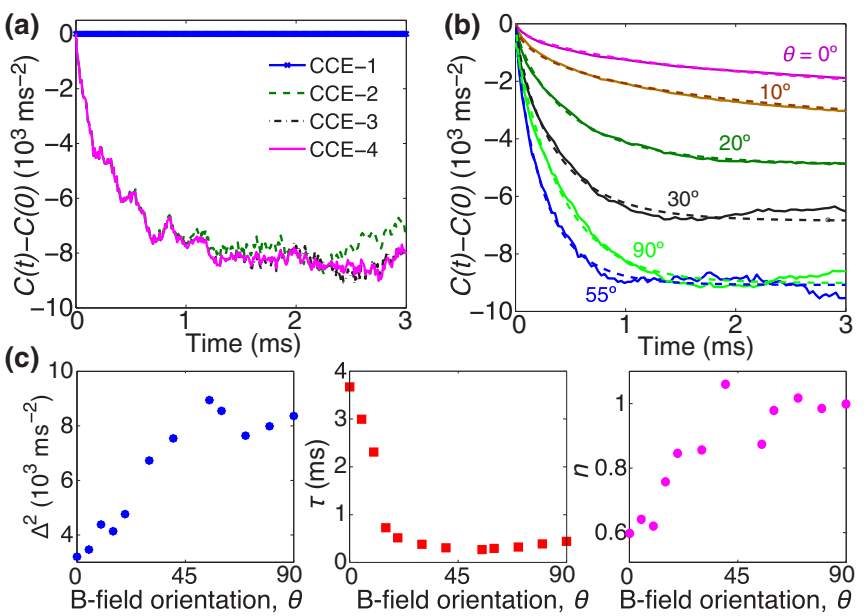

FIG. 1. (Color online) (a) Relative two-point correlation functions $C(t)-C(0)$ at the $\mathrm{CT}\left(B_{\mathrm{CT}}=79.9 \mathrm{mT}\right)$ calculated by different orders of CCE (CCE- $M$ denotes the $M$ th-order CCE truncation by keeping cluster correlations up to a certain size $M$ ). Here we choose a specific nuclear spin configuration with $\mathbf{B} \|[110]$ and the nuclear spin bath includes about $5000{ }^{29} \mathrm{Si}$ nuclear spins within $8 \mathrm{~nm}$ from the donor spin. (b) $C(t)-C(0)$ (solid lines) at the CT for several magnetic field orientations in the [001]-[110] plane with $\theta=0^{\circ}$ corresponding to [001]. Results are obtained by averaging over 50 different nuclear spin configurations. Dashed lines are fits of the form $\Delta^{2}\left\{\exp \left[-(|t| / \tau)^{n}\right]-1\right\}$. (c) The fitting parameters as functions of $\theta$.

As the major contribution [see Fig. 1(a) for the results of different CCE orders], the pairwise flip-flop processes in the nuclear spin bath lead to a correlation function as

$$
C(t)=C(0)+\sum_{\{i, j\}}\left\{\frac{2 Z_{i j}^{2} D_{i j}^{2}}{Z_{i j}^{2}+D_{i j}^{2}}\left[\cos \left(\omega_{i j} t\right)-1\right]\right\},
$$

where $\omega_{i j}=2 \sqrt{Z_{i j}^{2}+D_{i j}^{2}}$ is the noise frequency, $Z_{i j}=$ $\frac{\left|P_{+}\right|+\left|P_{-}\right|}{4}\left(A_{i}-A_{j}\right)$ is the energy cost of flip-flop processes, $D_{i j}=\gamma_{n}^{2}\left(3 \cos ^{2} \vartheta_{i j}-1\right) / 4\left|\mathbf{R}_{i j}\right|^{3}$ is the dipolar interaction strength, $\mathbf{R}_{i j}$ is the displacement between the $i$ th and $j$ th nuclear spins, and $\vartheta_{i j}$ is the angle between $\mathbf{R}_{i j}$ and $\mathbf{B}$. For a given $Z_{i j}$, the correlation amplitude and noise frequency increase with the dipolar interaction strength $D_{i j}$. When $\mathbf{B} \|$ [001], the nearest-neighbor nuclear spin pairs have zero dipolar interaction, so the flip-flop processes mainly occur between the second- and third-nearest neighbors. This produces a minimum in $\Delta$ and $n$ and maximum in $\tau$, as a result of the relatively slow nuclear spin dynamics. When B || [111], the nearest-neighbor nuclear spin pairs have the strongest dipolar interaction, so $\Delta$ reaches a maximum, $\tau$ a minimum, and $n \approx 1$ since the relatively fast nuclear spin dynamics make the noise like a classical Lorentzian noise.

Comparisons with experiments and quantum model. To explore the validity of the semiclassical model, we compare in Fig. 2 the decoherence obtained from Eq. (5) with the results from both the CCE method [8] and the experimental measurements. The measurements were conducted on a ${ }^{\text {nat }} \mathrm{Si}$ sample doped with ${ }^{209} \mathrm{Bi}$ at a concentration of $3 \times 10^{15} \mathrm{~cm}^{-3}$. The experiments were realized at $4.8 \mathrm{~K}$, which gives an electron spin relaxation time $T_{1 e}$ of $3.5 \mathrm{~s}$. The magnetic field (a) $B_{\mathrm{CT}}+0.15 \mathrm{mT}$

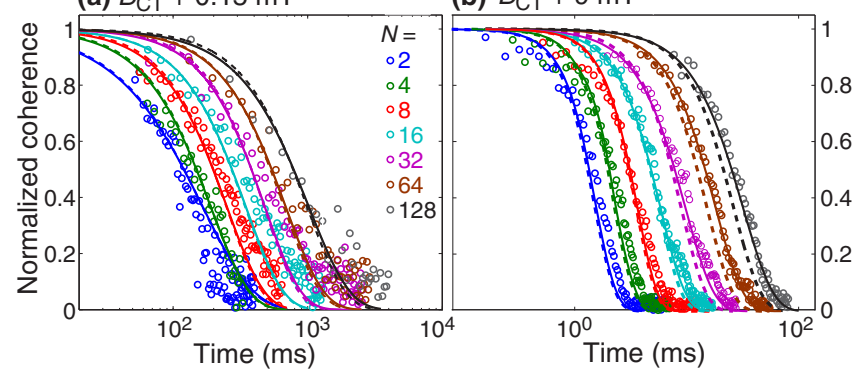

(c) $B_{\mathrm{CT}}+35 \mathrm{mT}$
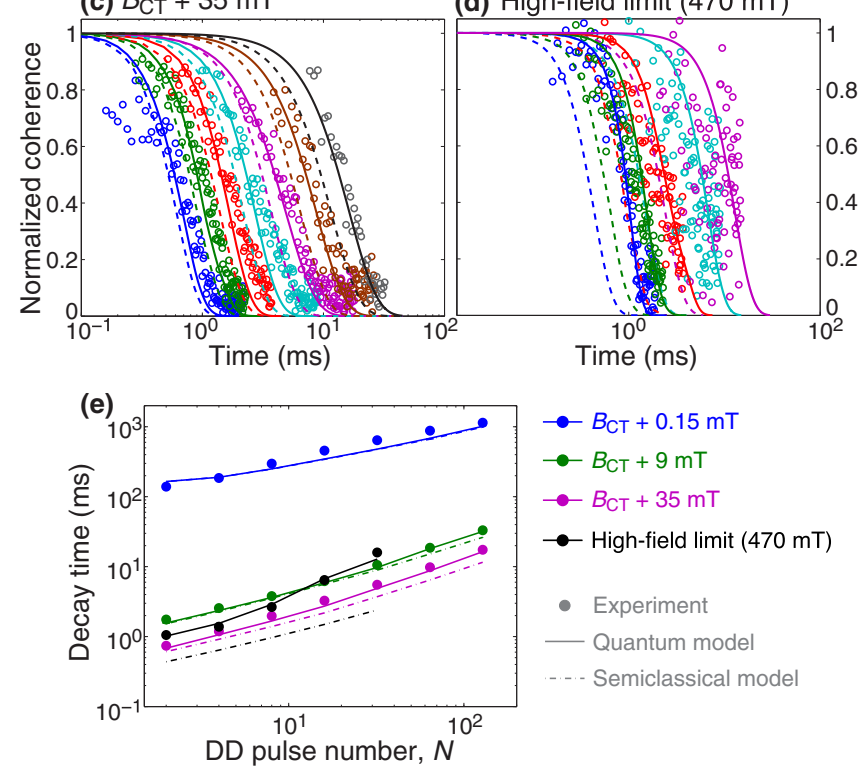

FIG. 2. (Color online) Comparisons of electron spin decoherence obtained by the quantum model (solid lines), the semiclassical model (dashed lines), and the experimental measurement (circles). Measurements were made using [(a)-(c)] the $|5,-1\rangle \leftrightarrow|4,-2\rangle$ transition at various fields shifted from the CT at $79.9 \mathrm{mT}$, or (d) the $|5,-4\rangle \leftrightarrow|4,-5\rangle$ transition close to the high-field limit at $468.65 \mathrm{mT}$. (e) The spin decoherence time for $T_{2 e}$ under CPMG control obtained from the models and experiment for various fields. Here $N=2,4,8,16,32,64,128$ corresponds to the DD control CPMG-2, XY-4, XY-8, XY-16, (XY-16) $\times 2,(X Y-16) \times 4,(X Y-16) \times 8$. In theoretical calculations, CPMG- $N$ is equivalent to $\mathrm{XY}-\mathrm{DD}$. The experimental data is corrected to remove effects of instantaneous diffusion and spin relaxation (Ref. [28]). The theoretical results are obtained by averaging over 100 nuclear spin configurations.

was aligned close to the [ $\overline{2} 41]$ crystal axis. The DD $\pi$ pulses were applied through adiabatic fast passages (hyperbolic secant functions, $20 \mu \mathrm{s}$ in duration spanning $6 \mathrm{MHz}$ close to the CT and $12 \mathrm{MHz}$ in the high-field limit) in order to achieve high-fidelity operations despite the electron spin resonance linewidth, which ranged from 6 to $12 \mathrm{MHz}$ at the fields studied here [28]. Owing to the high-fidelity DD control via the adiabatic passage method, remarkably, we managed to apply more than 100 control pulses and therefore extend the electron spin coherence times to $1 \mathrm{~s}$ in the natural silicon sample. We expect this can be further increased by using optimal magnetic field orientations and higher-order DD.

The results from the quantum model and the experimental data are in good agreement for all the magnetic fields. 
However, this is not the case for the semiclassical model. Close to the CT $\left[B_{\mathrm{CT}}+0.15 \mathrm{mT}\right.$ in Fig. 2(a), where $P_{+}=$ $\left.0.0527, P_{-}=0.0521\right]$, the semiclassical coherence, quantum coherence, and experimental results coincide for various DD control sequences, indicating that the nuclear spin bath is well described by a classical noise. It should be pointed out that the exact CT cannot be reached in the ensemble experiments since the hyperfine coupling to randomly oriented bath spins results in different donors requiring different values of $B$ in order to sit at a CT. Moreover, the CCE theory encounters difficulties at the exact CTs due to the dominance of long-range hyperfine-mediated nuclear spin interactions in the bath $[35,36]$. As the magnetic field is shifted away from the CT $\left[B_{\mathrm{CT}}+9 \mathrm{mT}\right.$ in Fig. 2(b), where $P_{+}=0.0695$, $\left.P_{-}=0.0340\right]$ the semiclassical model begins to show small deviations from the quantum model for DD with large numbers of pulses. Further still $\left[B_{\mathrm{CT}}+35 \mathrm{mT}\right.$ in Fig. 2(c), where $P_{+}=$ $0.1172, P_{-}=-0.0198$ ], there are clear deviations between the semiclassical and quantum models for all levels of DD control, but the time scales remain nearly equal. Finally, for the transitions in the high-field limit $[B=470 \mathrm{mT}$ in Fig. 2(d), where $\left.P_{+}=0.4264, P_{-}=-0.5\right]$, the semiclassical model ceases to be valid and shows significant differences from the quantum model and the experiment.

These comparisons demonstrate the classical Gaussian nature of the nuclear spin noise near the CT. This is understandable since the feedback of the central spin on the evolution of the nuclear spin bath $\left(\sim \frac{\left|P_{+}-P_{-}\right|}{2}\right)$ is largely reduced at the CT. For a bath with a large number of spins/particles, Gaussian statistics should be a good approximation (as a result of the central-limit theorem) [12]. However, our current study does not exclude the possibility of non-Gaussian statistics [12] causing the discrepancy between the classical noise model and the measurement in the regions far from the CT, which is an interesting issue for future study.

Limitations of DD noise spectroscopy method. Previous studies have adopted the DD noise spectroscopy method to characterize the baths [13-15]. The main idea is to use a specific DD control sequence (such as CPMG- $N$ with large $N$ ) with the filter function approximated as a Dirac delta function at $\omega_{0}= \pm \pi N / t$ [see Fig. 3(b)], i.e., $F(\omega, t) /\left(\omega^{2} t\right) \approx \pi[\delta(\omega-$ $\left.\left.\omega_{0}\right)+\delta\left(\omega+\omega_{0}\right)\right]$, and following Eq. (5) to determine the noise spectra as $S\left( \pm \omega_{0}\right)=-2 \ln [L(t)] /\left(t P_{e}^{2}\right)$. This method relies on the validity of the semiclassical Gaussian noise model.

However, the Gaussian noise model may be insufficient when the back-action of the central spin on the environment dynamics is significant. For example, in the ${ }^{\text {nat }} \mathrm{Si}: \mathrm{Bi}$ system, the Gaussian noise model is invalid for transitions far away from CTs. To demonstrate this point, we use the DD noise spectroscopy method to determine the effective noise spectra corresponding to the CPMG-100 case, and then use the derived noise spectra to calculate the spin decoherence under other DD control sequences. We note that a similar test has been carried out for the singlet-triplet spin qubit decoherence in semiconductor quantum dots [37]. In Fig. 3(a) we show the comparisons between the exact decoherence model and the semiclassical model using the DD noise spectroscopy method, and find increasing discrepancies as the pulse number of CPMG- $N$ deviates from 100. In contrast, close to the CTs, the DD noise spectroscopy method can not only reproduce the
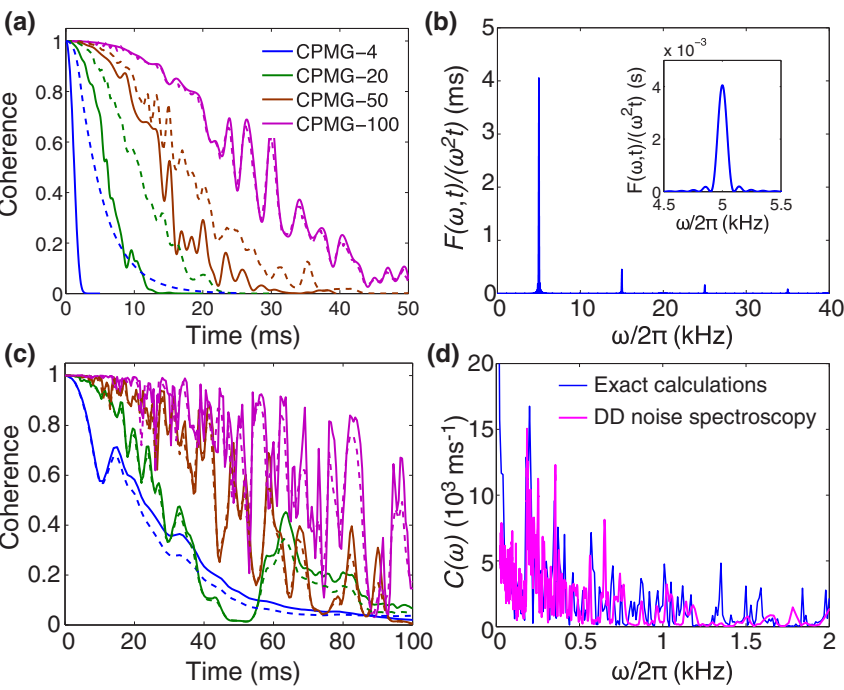

FIG. 3. (Color online) Calculated electron spin decoherence under an exact quantum model (solid lines) and semiclassical model obtained from noise spectroscopy of the CPMG-100 DD (dashed lines), evaluated (a) far from the $\mathrm{CT}\left(B_{\mathrm{CT}}+1000 \mathrm{G}\right)$ and (c) close to the CT $\left(B_{\mathrm{CT}}+10 \mathrm{G}\right)$. (b) Filter function $F(\omega, t) /\left(\omega^{2} t\right)$ for CPMG-100 noise spectroscopy, with $t=10 \mathrm{~ms}$. (d) Comparison of the noise spectrum from the CPMG-100 spectral decomposition in (c) to the exact one from CCE calculations. Here we choose a specific nuclear spin configuration with $\mathbf{B} \|[110]$.

spin decoherence curves for other DD control [see Fig. 3(c)], but also well reproduce the exact noise spectrum obtained from CCE calculations [see Fig. 3(d)]. Here the decoherence profile shows increasingly violent oscillations (corresponding to the relatively high-frequency noise) for a single nuclear spin configuration, reducing the efficiency of DD control at long-time scales. The reason is that when the DD modulation frequency matches the noise frequency, the noise can be amplified rather than suppressed [38].

Summary. We have presented a semiclassical model to study the decoherence of electron spin qubits in natural silicon near the CTs in a ${ }^{\text {nat }} \mathrm{Si}: \mathrm{Bi}$ system. The comparisons of the semiclassical results against the exact quantum results and experimental measurements demonstrate that the nuclear spin bath acts more and more like a classical Gaussian noise as the CTs are approached. Our findings deepen the understanding of spin baths near CTs and are useful for optimizing the DD control in silicon-based quantum computation-indeed we have already shown here that using DD at CTs, electron spin coherence times of about $1 \mathrm{~s}$ can be measured in natural silicon.

Acknowledgments. This work was supported by National Basic Research Program of China (973 Program) under Grant No.G2009CB929300, National Natural Science Foundation of China under Grant No.61121491, and Hong Kong RGC/CRF CUHK4/CRF/12G. Work at UCL was supported by the European Research Council under the European Community's Seventh Framework Programme (FP7/2007-2013)/ERC (Grant No.279781), and by the Engineering and Physical Sciences Research Council (EPSRC) Grants No. EP/K025945/1 and No. EP/I035536/2. J.J.L.M. is supported by the Royal Society.

W.-L.M. and G.W. contributed equally to this work. 
[1] W. H. Zurek, Rev. Mod. Phys. 75, 715 (2003).

[2] W. H. Zurek, Nat. Phys. 5, 181 (2009).

[3] T. D. Ladd, F. Jelezko, R. Laflamme, Y. Nakamura, C. Monroe, and J. L. O'Brien, Nature (London) 464, 45 (2010).

[4] I. A. Merkulov, Al. L. Efros, and M. Rosen, Phys. Rev. B 65, 205309 (2002).

[5] R. de Sousa and S. Das Sarma, Phys. Rev. B 67, 033301 (2003).

[6] W. M. Witzel and S. Das Sarma, Phys. Rev. B 74, 035322 (2006).

[7] W. Yao, R.-B. Liu, and L. J. Sham, Phys. Rev. B 74, 195301 (2006).

[8] W. Yang and R.-B. Liu, Phys. Rev. B 78, 085315 (2008).

[9] J. R. Maze, J. M. Taylor, and M. D. Lukin, Phys. Rev. B 78, 094303 (2008).

[10] N. Zhao, Z.-Y. Wang, and R.-B. Liu, Phys. Rev. Lett. 106, 217205 (2011).

[11] P. Huang, X. Kong, N. Zhao, F. Shi, P. Wang, X. Rong, R.-B. Liu, and J. Du, Nat. Commun. 2, 570 (2011).

[12] W. M. Witzel, K. Young, and S. Das Sarma, Phys. Rev. B 90, 115431 (2014).

[13] G. A. Álvarez and D. Suter, Phys. Rev. Lett. 107, 230501 (2011).

[14] N. Bar-Gill, L. M. Pham, C. Belthangady, D. Le Sage, P. Cappellaro, J. R. Maze, M. D. Lukin, A. Yacoby, and R. Walsworth, Nat. Commun. 3, 858 (2012).

[15] J. Bylander, S. Gustavsson, F. Yan, F. Yoshihara, K. Harrabi, G. Fitch, D. G. Cory, Y. Nakamura, J.-S. Tsai, and W. D. Oliver, Nat. Phys. 7, 565 (2011).

[16] Ł. Cywiński, Phys. Rev. A 90, 042307 (2014).

[17] J. T. Muhonen et al., Nat. Nanotechnol. 9, 986 (2014).

[18] R. E. George, W. Witzel, H. Riemann, N. V. Abrosimov, N. Notzel, M. L. W. Thewalt, and J. J. L. Morton, Phys. Rev. Lett. 105, 067601 (2010)

[19] G. W. Morley, M. Warner, A. M. Stoneham, P. T. Greenland, J. van Tol, C. W. M. Kay, and G. Aeppli, Nat. Mater. 9, 725 (2010).

[20] M. H. Mohammady, G. W. Morley, A. Nazir, and T. S. Monteiro, Phys. Rev. B 85, 094404 (2012).
[21] G. Wolfowicz et al., Phys. Rev. B 86, 245301 (2012).

[22] G. Wolfowicz, A. M. Tyryshkin, R. E. George, H. Riemann, N. V. Abrosimov, P. Becker, H.-J. Pohl, M. L. W. Thewalt, S. A. Lyon, and J. J. L. Morton, Nat. Nanotechnol. 8, 561 (2013).

[23] M. H. Mohammady, G. W. Morley, and T. S. Monteiro, Phys. Rev. Lett. 105, 067602 (2010).

[24] S. J. Balian, G. Wolfowicz, J. J. L. Morton, and T. S. Monteiro, Phys. Rev. B 89, 045403 (2014).

[25] S. J. Balian, M. B. A. Kunze, M. H. Mohammady, G. W. Morley, W. M. Witzel, C. W. M. Kay, and T. S. Monteiro, Phys. Rev. B 86, 104428 (2012).

[26] R. de Sousa and S. Das Sarma, Phys. Rev. B 68, 115322 (2003).

[27] Ł. Cywiński, R. M. Lutchyn, C. P. Nave, and S. Das Sarma, Phys. Rev. B 77, 174509 (2008).

[28] See Supplemental Material at http://link.aps.org/supplemental/ 10.1103/PhysRevB.92.161403, which includes Refs. [6$8,18,21,22]$, for detailed descriptions of the calculations and experimental setup.

[29] L. Viola, E. Knill, and S. Lloyd, Phys. Rev. Lett. 82, 2417 (1999).

[30] G. de Lange, Z. H. Wang, D. Risté, V. V. Dobrovitski, and R. Hanson, Science 330, 60 (2010).

[31] H. Y. Carr and E. M. Purcell, Phys. Rev. 94, 630 (1954).

[32] S. Meiboom and D. Gill, Rev. Sci. Instrum. 29, 688 (1958).

[33] R. de Sousa, Top. Appl. Phys. 115, 183 (2009).

[34] A. M. Tyryshkin, J. J. L. Morton, S. C. Benjamin, A. Ardavan, G. A. D. Briggs, J. W. Ager, and S. A. Lyon, J. Phys.: Condens. Matter 18, S783 (2006).

[35] Ł. Cywiński, W. M. Witzel, and S. Das Sarma, Phys. Rev. Lett. 102, 057601 (2009).

[36] Ł. Cywiński, W. M. Witzel, and S. Das Sarma, Phys. Rev. B 79, 245314 (2009).

[37] J. Medford, L. Cywiński, C. Barthel, C. M. Marcus, M. P. Hanson, and A. C. Gossard, Phys. Rev. Lett. 108, 086802 (2012).

[38] N. Zhao, J.-L. Hu, S.-W. Ho, T. K. Wan, and R. B. Liu, Nat. Nanotechnol. 6, 242 (2011). 